\title{
Internal surface measurement of metal powder bed fusion parts
}

\author{
Adam Thompson ${ }^{1}$, Nicola Senin ${ }^{1,2}$, Ian Maskery ${ }^{3}$, Lars Körner ${ }^{1}$, Simon Lawes ${ }^{1}$, \\ Richard Leach ${ }^{1}$ \\ ${ }^{1}$ Manufacturing Metrology Team, Faculty of Engineering, University of Nottingham, \\ NG7 2RD, UK \\ ${ }^{2}$ Department of Engineering, University of Perugia, 06125, Italy \\ ${ }^{3}$ Centre for Additive Manufacturing, Faculty of Engineering, University of Nottingham, \\ NG7 2RD, UK
}

\begin{abstract}
Recent advances in X-ray computed tomography (XCT) have allowed for measurement resolutions approaching the point where XCT can be used for measuring surface topography. These advances make XCT appealing for measuring hard-to-reach or internal surfaces, such as those often present in additively manufactured parts. To demonstrate the feasibility and potential of XCT for topography measurement, topography datasets obtained using two XCT systems are compared to those acquired using coherence scanning interferometry and focus variation microscopy. A hollow Ti6Al4V part produced by laser powder bed fusion is used as a measurement artefact. The artefact comprises two component halves that can be separated to expose the internal surfaces. Measured surface datasets are accurately aligned and similarly cropped, and compared by various qualitative and quantitative means, including the computation of ISO $25178-2$ areal surface texture parameters, commonly used in part quality assessment. Results show that XCT can non-destructively provide surface information comparable with more conventional surface measurement technologies, thus representing a viable alternative to more conventional measurement, particularly appealing for hard-to-reach and internal surfaces.
\end{abstract}

Key words: X-ray computed tomography, metrology, surface texture, metal powder bed fusion

\section{Introduction}

Additive manufacturing (AM) technologies have the ability to produce parts containing complex geometries that were previously impossible to manufacture by conventional means [1]. A number of barriers to increased adoption of AM parts exist, however, relating to the difficulties in applying quality assurance principles, such as dimensional and geometric inspection and verification [2]. In particular, when inspecting AM surfaces, conventional optical and contact surface measurement solutions are often incapable of measuring the inaccessible and internal surfaces. Such conditions are common with typical AM geometries, such as hollow parts and lattice structures [3-5].

X-ray computed tomography (XCT) has recently become established as a useful tool in holistic measurement of industrial parts, and is steadily being incorporated into the metrological toolbox [6]. Although much work remains in standardisation of the use of XCT for metrology (ISO 10360-11 [7] is still in the draft stages), XCT has begun to show promise for the verification of internal geometries present in AM parts [8]. Although the spatial resolutions typically achievable by XCT have not historically been at the level required to capture the smaller-scale formations of a surface in addition to the overall shape, advanced systems are approaching these resolutions in their best-case measurement scenarios, and so XCT is becoming an viable option for measurement of surface topography. When 
considering the fact that AM parts commonly feature complex, internal geometries, the prospect of using $\mathrm{XCT}$ for surface topography measurement appeals further, as a method of overcoming the access requirement problems intrinsic to contact and optical measurements. The use of XCT for surface topography measurement is highlighted in a number of recent studies [9-14]. Specifically, Pyka et al. $[9,10]$ performed the first surface topography measurement using XCT, by extracting profiles from slice data obtained from XCT measurement of lattice struts. Townsend et al. $[12,13,15]$ and Thompson et al. [14] extended this work by initiating a more extensive examination of XCT topography measurement performance in comparison to conventional optical surface measurements, with the most recent work by Townsend et al. [13] examining the output of a number of measurements performed across several laboratories. Much work exists in the validation of XCT for internal topography measurement. However, to date and to the authors' knowledge, no research effort has been specifically dedicated to investigating the challenges of measuring topography of internal surfaces. To address this research need, an investigation comparing internal XCT surface measurements and measurements made using conventional optical surface technologies is presented in this paper.

\section{Methods}

A metal laser powder bed fusion (LPBF) hollow artefact was measured using two commercial XCT systems (labelled "XCT 1" and "XCT 2" respectively), as well as by two non-contact optical measurement systems. Measurements were performed using input parameters optimised for each system based upon the manufacturer's recommendations and the authors' prior experience, but all instrument names have been redacted from this publication to prevent undue comparison of commercial instruments. The artefact used in this work was produced in two separable parts (see figure 1) from Ti6A14V using an EOSINT M 280 metal LPBF machine. The manufacturer's proprietary process parameters for Ti6A14V were used to produce the artefact. Ti6Al4V was chosen as the artefact material for its suitability to XCT measurement [8] and general industrial relevance. When assembled, the artefact simulates the metrological challenge of internal geometries as surfaces become inaccessible to conventional optical surface measurement solutions. When separated, surfaces can be inspected using optical technologies.

a)

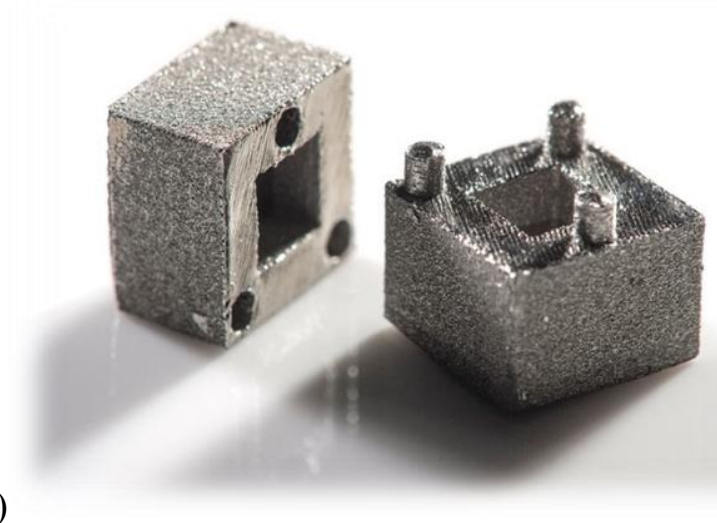

b)

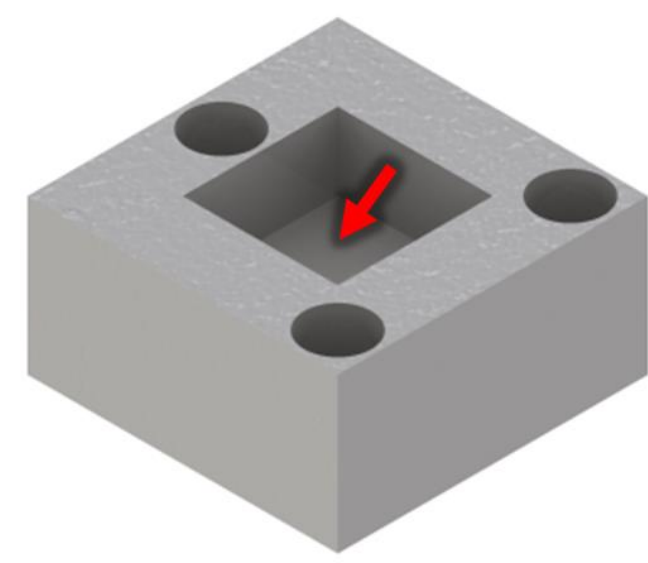

Figure 1. a) Artefact for the measurement of internal surface texture. When assembled, cube dimensions are $(10 \times 10 \times 10) \mathrm{mm} ; b)$ The surface of interest, indicated by the arrow on a CAD rendering of one half of the artefact.

\subsection{XCT measurements of surface topography}

XCT 1 measurement setup: voltage $150 \mathrm{kV}$, current $36 \mu \mathrm{A}, 3142$ projections formed from averaging two images per projection, each using an exposure of $2829 \mathrm{~ms}$, geometric magnification $35 \times$ yielding a voxel size of $5.7 \mu \mathrm{m}$ after reconstruction. A warmup scan of approximately one hour was performed 
prior to the scan and a $0.25 \mathrm{~mm}$ copper pre-filter was used between the X-ray source and the specimen. $\mathrm{X}$-ray imaging and volumetric reconstruction were performed using manufacturer's proprietary software, using filtered back projection with a beam hardening correction and a Hanning noise filter [16].

XCT 2 measurement setup: voltage $160 \mathrm{kV}$, current $63 \mu \mathrm{A}, 1600$ projections formed from one image per projection, each using an exposure of $6000 \mathrm{~ms}$, geometric magnification of 5.75× and optical magnification of $0.4 \times$ yielding a voxel size of $5 \mu \mathrm{m}$ after reconstruction. A proprietary pre-filter was used according to the manufacturer's guidelines. X-ray imaging and volumetric reconstruction were performed using the manufacturer's proprietary software using filtered back projection with a beam hardening correction and a Gaussian reconstruction filter with a kernel size of 1.

Reconstructed volumetric data were imported into VolumeGraphics VGStudioMAX 3.0 [17] and surfaces were determined using the iterative maximum gradient method over four voxels, using the ISO50 isosurface as a starting point (see figure 2a) [18].

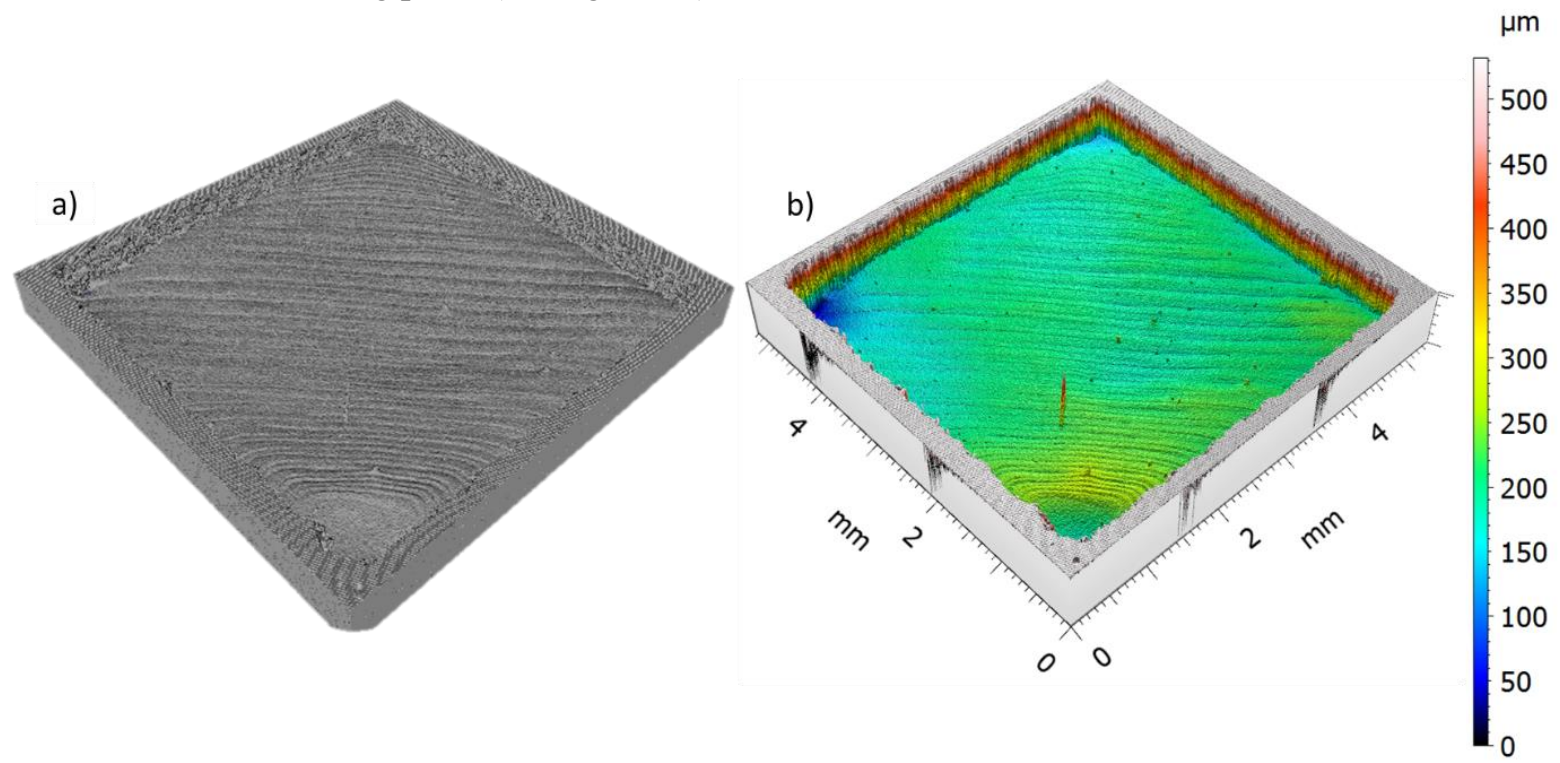

Figure 2. Surface determined for XCT 1 data: a) rendered in MeshLab [19]; b) resampled into a height map and rendered in MountainsMap.

\subsection{Optical measurements of surface topography}

Coherence scanning interferometry (CSI) measurement setup (where LR is lateral resolution and FoV is field of view): $20 \times$ objective lens at $1 \times$ zoom (NA 0.40, FoV $0.42 \mathrm{~mm} \times 0.42 \mathrm{~mm}$, LR-pixel $0.41 \mu \mathrm{m}$, LR-optical $0.68 \mu \mathrm{m})$. Stitching of multiple FoVs was performed using the manufacturer's proprietary software. Vertical stitching was also applied, to merge two measurement $z$ intervals $(145 \mu \mathrm{m}$ and $100 \mu \mathrm{m}$ wide respectively, with $10 \mu \mathrm{m}$ overlap). LR-pixel refers to the pixel width of the detector used by each instrument, while LR-optical refers the calculated optical Sparrow limit of each instrument.

Focus variation (FV) measurement setup: $20 \times$ objective lens (NA 0.40, FoV $0.81 \mathrm{~mm} \times 0.81 \mathrm{~mm}$, LRpixel $0.44 \mu \mathrm{m}$, LR-optical $0.68 \mu \mathrm{m}$ ) was used with ring light illumination. Vertical resolution was set at $50 \mathrm{~nm}$ and LR-contrast at $3 \mu \mathrm{m}$, where LR-contrast refers specifically to the distance from the centre of each pixel used by the FV instrument to compute local contrast; selected during the measurement. Stitching of multiple fields of view was performed in the manufacturers' proprietary software. 


\subsection{Data processing}

XCT surface data were cropped to extract the surface of interest in VGStudioMAX, and exported as triangulated meshes in .stl format. Triangulated meshes were rotated in MeshLab [19] to align the surface normal to the $z$ axis (surface normal computed via principal component analysis [20] on the mesh point cloud), and exported again as an .stl. The rotated mesh was then imported into the surface metrology software MountainsMap [21] and resampled into height maps at a resolution automatically determined by MountainsMap to match the point density of the triangulated mesh (see figure $2 b$ ).

Height maps obtained by XCT and optical measurement were relocated in the same coordinate system using MountainsMap by application of a marker-based coarse alignment, followed by cross-correlation based global algorithmic alignment [22]. From the aligned height maps, regions of size $(1.5 \times 1.5) \mathrm{mm}$ were extracted, and levelled by least-squares mean plane subtraction, allowing like-for-like comparison of surface data. This sample size was chosen as, at $20 \times$ magnification, measurement of a larger area by CSI was deemed unfeasible due to the prohibitive number of stitching operations, and measurement times. Topography datasets were bandwidth-matched [23] (involving the application of filtering operations with identical cut-off wavelengths across datasets) to allow comparison of the resulting parameters. Extracted surfaces were initially filtered using a Gaussian convolution S-filter with a $13 \mu \mathrm{m}$ cut-off to remove small-scale surface features; chosen as the minimum possible for the lowest lateral resolution height map (XCT 2), representative of a grid of $4 \times 4$ pixels. A Gaussian convolution L-filter with a $1.5 \mathrm{~mm}$ cut-off was then chosen as equal to the size of the region of interest. This operator was applied to remove tilt and waviness at scales larger than the field of view, therefore obtaining SF surfaces. A Gaussian convolution L-filter with a $0.5 \mathrm{~mm}$ cut-off was applied to remove smaller scale waviness; thus obtaining SL surfaces. This cut-off was chosen based upon visual inspection of the surface in question. SL surfaces were then truncated to remove attached particles detected in the measurement, by voiding height values above a value based on visual inspection of each surface. ISO 25178-2 [24,25] areal texture parameters were calculated for SF, SL and truncated SL surfaces. Additionally, analyses of texture direction and power spectral density were performed.

The bandwidth matching process applied to obtain the SL surfaces is designed to remove spatial wavelengths that are not captured by all the measurement technologies, thus homogenising the spatial measurement bandwidths of the examined data. This process allows for comparability between datasets regardless of differences between the resolutions of the raw data. However, the bandwidth matching process intrinsically involves the disposal of information present in some of the datasets (those covering a wider range of scales), which is why the SF surfaces (i.e. the original datasets with no removed wavelengths) have also been considered in the analysis. The most notable difference between the investigated measurement technologies (in terms of their contents) is observed at the shortest wavelengths (largest spatial frequencies) because of the intrinsically higher spatial resolution achievable using optical techniques as opposed to XCT. However, specifically regarding metal AM surfaces, in recent work by the authors (Senin et al. [26]) it was demonstrated that many interesting LPBF surface topography features are accurately represented within the bandwidths shared by all the compared technologies.

\section{Results and discussion}

\subsection{Comparison of surface topography features}

Visual comparison was performed on reconstructed top views of the SF height maps (see figure 3). For visualisation, false colours proportional to heights were used in the reconstructions. Colour scales were homogenised by truncating height points above and below a common reference vertical range. 
Truncation was applied in figure 3 to homogenise colour scales for visualisation purposes only, while the original datasets were maintained for quantitative comparison.

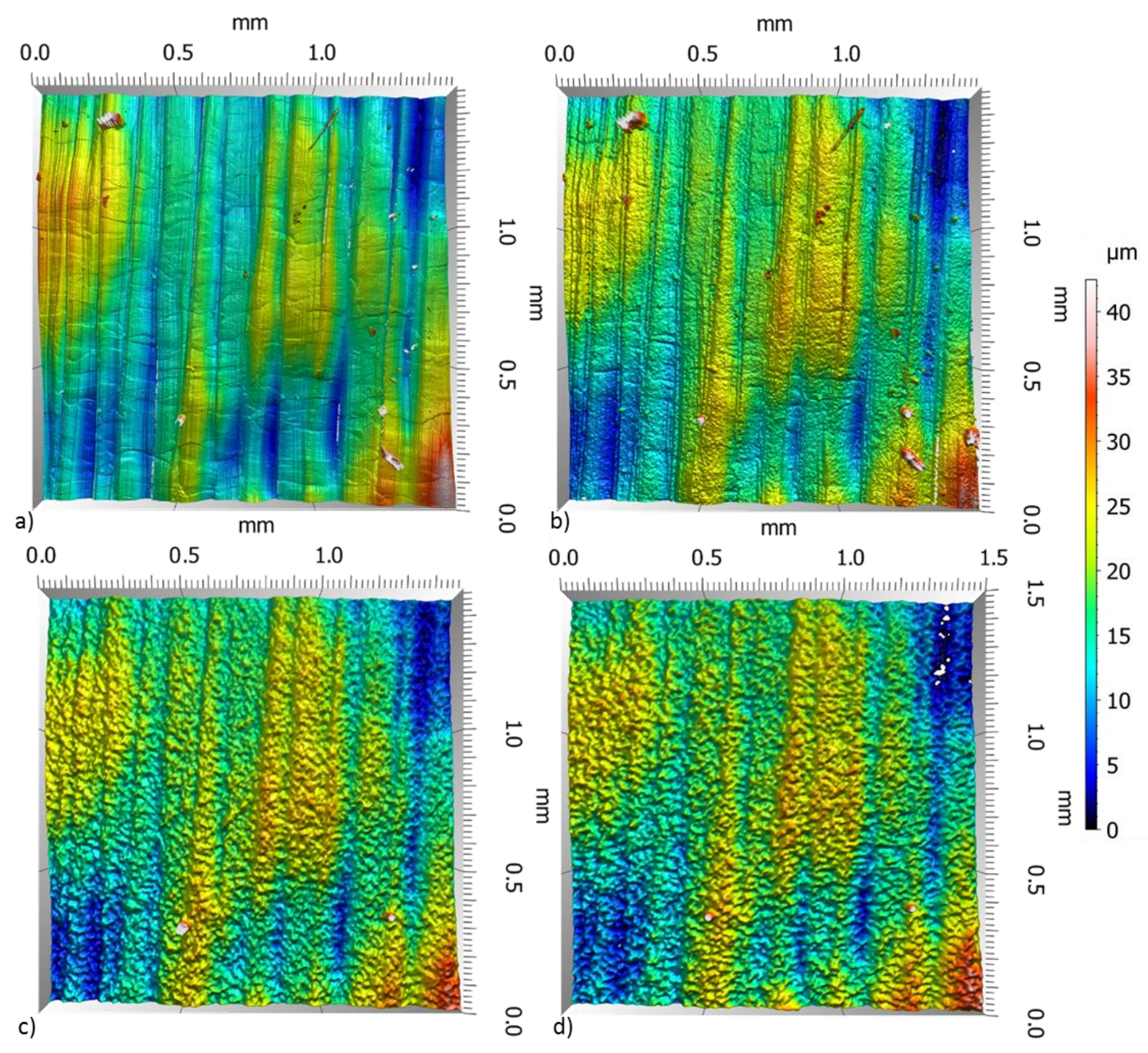

Figure 3. Levelled and truncated surface height maps: a) CSI; b) FV; c) XCT 1; d) XCT 2. Visual similarity is seen between datasets, though some smaller features present in optical data are not all present in XCT data (e.g. particles).

Visual investigation reveals notable similarities between all datasets, in that all topographies feature a visibly similar reconstruction of weld tracks features and of larger-scale waviness components. Reconstruction of smaller-scale features, however, varies greatly between datasets. Optical reconstructions are similar, though $\mathrm{FV}$ data shows high-spatial frequency noise of greater magnitude and volume that CSI data. However, both XCT systems return noticeably larger amounts of high spatial frequency noise when compared to optical measurement.

\subsection{Comparison of areal texture parameters}

ISO 25178-2 [24,25] areal texture parameters calculated for the data displayed in figure 3 are presented respectively in tables 1, 2 and 3, for SF, SL and truncated SL surfaces. Only one region was analysed per surface type, leading to only one parameter value per measurement. The reported parameter values 
are, therefore, only indicative of the differences between the investigated datasets, and cannot be considered statistically significant indicators of overall performance of one measurement solution compared to another. Nevertheless, this can provide an indicator of the ability of XCT to measure surface topography.

Six commonly used ISO 25178-2 [24,25] surface texture parameters are considered here: the arithmetical mean height of the scale-limited surface $S a$ (the surface allegory of the most commonly used profile parameter $R a$ [5]); the root mean square height of the scale-limited surface $S q$; the skewness of the scale-limited surface Ssk (a measure of the amount of material above or below the mean surface); the kurtosis of the scale-limited surface $S k u$ (a measure of the spikiness of the distribution of heights); the texture direction of the scale-limited surface $S t d$; and the autocorrelation length Sal (the minimum horizontal distance in which the auto-correlation function decays to 0.2 ).

First, parameters computed for SF surfaces are examined (see table 1). For the SF surfaces, the optical techniques return $S a$ and $S q$ parameters that are the most similar to one another, which is to be expected, as both technologies are well established topographical measurement solutions. XCT instruments also return similar $S a$ and $S q$ parameters, consistent with the results of visual observation of the reconstructed topographies (see figure 3). Large differences are seen between instruments for Ssk and $S k u$ parameters, while similar values are returned for the Sal parameters. Consistency between Std parameters can be considered an indicator of the quality of the alignment of surfaces.

Table 1. ISO 25178-2 [24,25] surface parameters for SF surfaces

\begin{tabular}{|c|c|c|c|c|}
\hline Parameter & CSI & $\mathrm{FV}$ & XCT 1 & XCT 2 \\
\hline $\mathrm{Sa} / \mu \mathrm{m}$ & 3.33 & 3.33 & 3.30 & 3.47 \\
\hline$S q / \mu \mathrm{m}$ & 4.25 & 4.37 & 4.30 & 4.36 \\
\hline Ssk & 0.88 & 0.94 & 1.25 & 0.15 \\
\hline Sku & 7.31 & 8.45 & 18.7 & 3.31 \\
\hline$S t d /^{\circ}$ & 85.8 & 86.0 & 85.8 & 85.7 \\
\hline $\mathrm{Sal} / \mathrm{mm}$ & 0.108 & 0.104 & 0.113 & 0.116 \\
\hline
\end{tabular}

Following assessment of SF surfaces, parameters computed from SL surfaces were examined. For SL surfaces, all instruments again returned similar $S a$ and $S q$ parameters, though the effect of the L-filter in this case appears to intensify differences between calculated $S a$ parameters, while slightly reducing differences between calculated $S q$ parameters. Differences between instruments for $S s k$ and $S k u$ parameters are exacerbated by the L-filter, while reducing differences for Sal and Std parameters.

Table 2. ISO 25178-2 [24,25] surface parameters for SL surfaces

\begin{tabular}{lllll}
\hline Parameter & CSI & FV & XCT 1 & XCT 2 \\
\hline$S a / \mu \mathrm{m}$ & 1.96 & 1.91 & 1.94 & 2.09 \\
$S q / \mu \mathrm{m}$ & 2.67 & 2.72 & 2.67 & 2.68 \\
$S s k$ & 1.52 & 2.20 & 3.02 & 0.08 \\
$S k u$ & 22.4 & 27.6 & 66.0 & 5.18 \\
$\mathrm{Std} /{ }^{\circ}$ & 85.8 & 85.8 & 85.8 & 85.7 \\
$\mathrm{Sal} / \mathrm{mm}$ & 0.0415 & 0.0450 & 0.0390 & 0.0409 \\
\hline
\end{tabular}

The most notable parameter differences in both of the aforementioned cases occur for Ssk and Sku parameters, with results varying greatly between instruments and filtering conditions. Calculations of $S s k$ and $S k u$ parameters involve summation of higher order powers of surface height values than those for $S a$ and $S q$ [25] and as such are more heavily affected by the presence of outliers in the data. As part of preliminary investigations, a simple outlier removal operation was performed, but yielded no noticeable difference to the calculated parameters. However, further visual examination of raw and 
filtered surface data reveals that the instruments show different responses to exogenous particles, (i.e. particles attached to the surface), a common feature of parts produced by LBPF. Specifically, a number of attached particles within the region of interest appear clearly in data acquired by the optical systems, but do not appear at all in XCT data (see figure 3). These particles sit above the surface, and due to the outlier-sensitive natures of the $S s k$ and $S k u$ parameters, could have a substantial effect on calculated parameters. This 'missing particles' phenomena is visualised in figure 4, where CSI data (figure 4a) contains three exogenous particles, while the algorithmically-determined XCT surfaces show only two particles (XCT1 data is shown as an example in figure 4b). While figure 4a was generated from CSI data, the missing particle is similarly present in FV data. While it is possible that such a particle could have been removed/deposited between measurements, and so could be missing in one dataset and present in another, further examination of XCT data in this case provides more information. If a surface is determined from XCT data through the use of thresholding, as opposed to by using the iterative algorithm (as exemplified by figure $4 \mathrm{c}$ and $4 \mathrm{~d}$ ), the missing particle (bottom right of each image) can be seen, while the particle visible to the left of figures $4 \mathrm{a}$ and $4 \mathrm{~b}$ disappears. In the thresholding case, however, the surface is greatly degraded by the presence of increased noise, and other particles may become indiscernible from noise for this surface (as is the case for the leftmost particle present in this data). This effect is most likely as a result of differing material properties (i.e. X-ray attenuation) in the particle in question, resulting in errors in the algorithmically-determined surface.
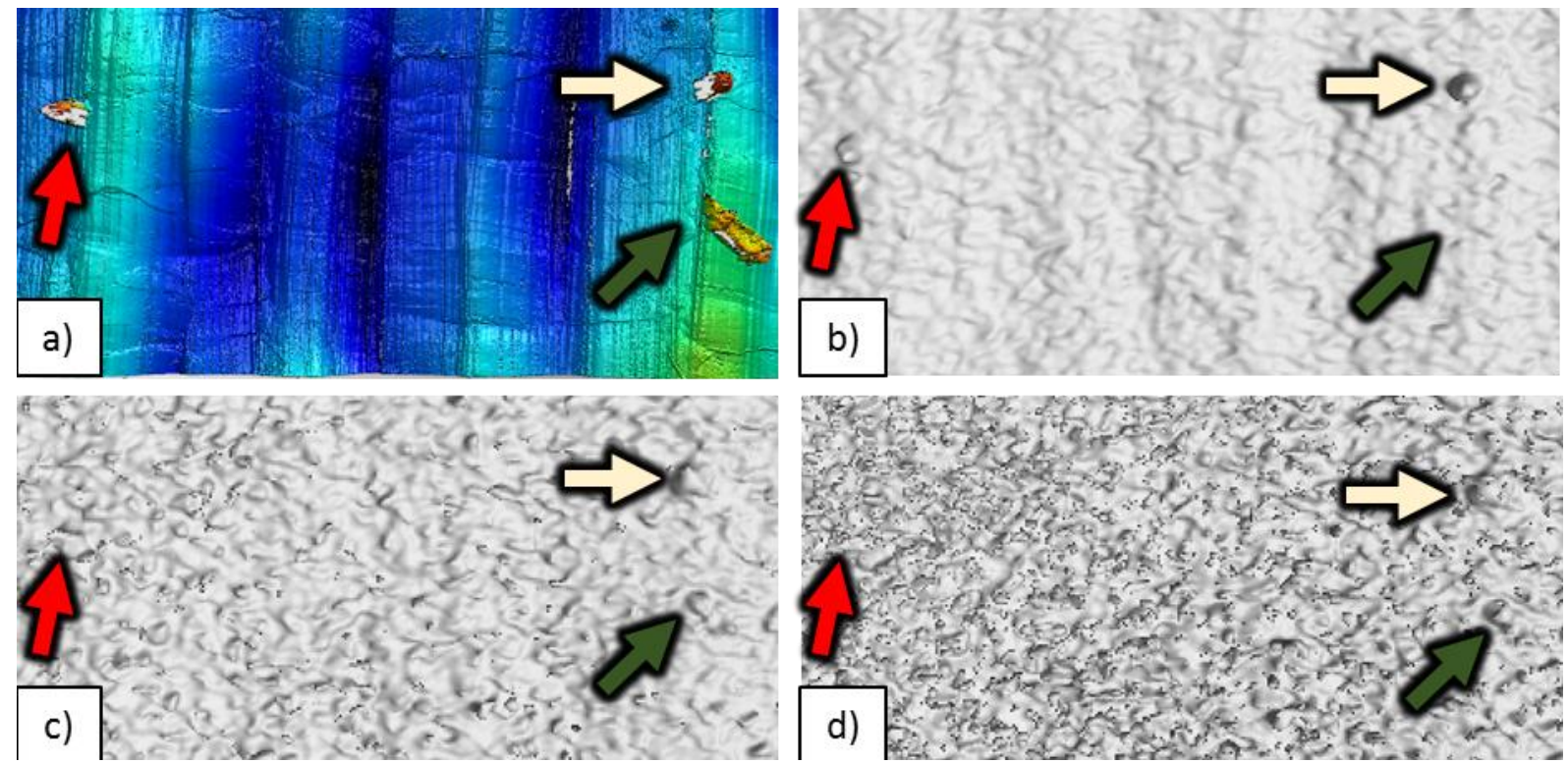

Figure 4. Comparison of particles present on a portion of the surface with differing surface determination: a) CSI reference (rendered in MountainsMap); b) XCT 1 using gradient based iterative algorithmic surface determination; c) XCT 1 using ISO50 grey value based surface; d) XCT 1 using manually chosen grey value based surface (XCT surfaces rendered in VGStudioMAX).

To account for the presence of attached particles on the measured surfaces and differences between datasets, data were truncated to remove particles from the measured data and parameters recalculated (reported in table 3). Following truncation, calculated Ssk and Sku parameters are much closer between instruments, and notably the sign of the $S s k$ parameter changes compared to the case where particles are not removed from the data. It is clear that attached particles have a substantial effect on these higherorder parameters, and in good practice, care should be taken in the process of gaining a reliable XCT surface measurement as such particles can clearly be missed by the process. Lower order $S a$ and $S q$ parameters are changed by attached particle removal and discrepancies are slightly exacerbated, but the resultant parameters are less affected by the presence of attached particles. It is also of note that, in order 
to gain reliable $S s k$ and $S k u$ parameters from LPBF surfaces, an attached particle removal operation is recommended.

Table 3. ISO 25178-2 [24,25] surface parameters for truncated SL surfaces

\begin{tabular}{lllll}
\hline Parameter & CSI & FV & XCT 1 & XCT 2 \\
\hline$S a / \mu \mathrm{m}$ & 1.92 & 1.83 & 1.91 & 2.08 \\
$S q / \mu \mathrm{m}$ & 2.42 & 2.32 & 2.41 & 2.64 \\
$S s k$ & -0.330 & -0.289 & -0.301 & -0.175 \\
$S k u$ & 3.12 & 3.36 & 3.20 & 3.20 \\
$\mathrm{Std} /{ }^{\circ}$ & 85.8 & 85.8 & 85.7 & 85.7 \\
$\mathrm{Sal} / \mathrm{mm}$ & 0.0422 & 0.0459 & 0.0406 & 0.0410 \\
\hline
\end{tabular}

Although Std parameters are consistent between datasets in all cases, surface texture direction analysis (see figure 5) reveals more information than the parameters can alone, through further analysis of the directional attributes of features present in the XCT and optical datasets. Each plot represents the values of the angular power spectra for the SL surfaces as a function of surface direction. The angle corresponding to the maximum value is taken as Std. Direction analyses show that, while the position of the primary peak is consistent between spectra, the ratio between the size of the primary peak and the smaller peaks (i.e. the signal to noise ratio) varies. This ratio is greatest in the CSI data and smallest in XCT 2 data, while the FV ratio is slightly greater than the CSI ratio, and the XCT 1 ratio is slightly lower than the XCT 2 ratio. As measurement noise is in this case random and, therefore, devoid of direction, this ratio is attributed to greater noise in XCT measurements than in optical datasets. It is clear that the values of the angular power spectrum are generally higher in multiple directions in the case of the noisier XCT datasets, making it more difficult to isolate the highest peak. Despite the increased noise, isolation of this peak is possible in both of these XCT cases, and improvement is likely possible with further optimisation of XCT acquisitions. However, in the case of a noisier XCT dataset, it is likely that such isolation may not similarly be possible. 

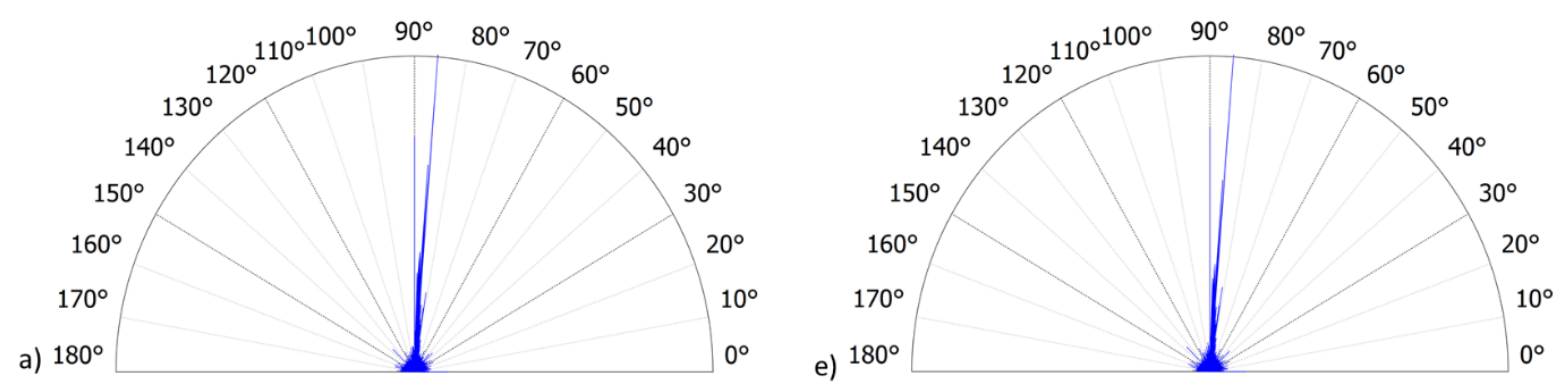

b) $180^{\circ}$
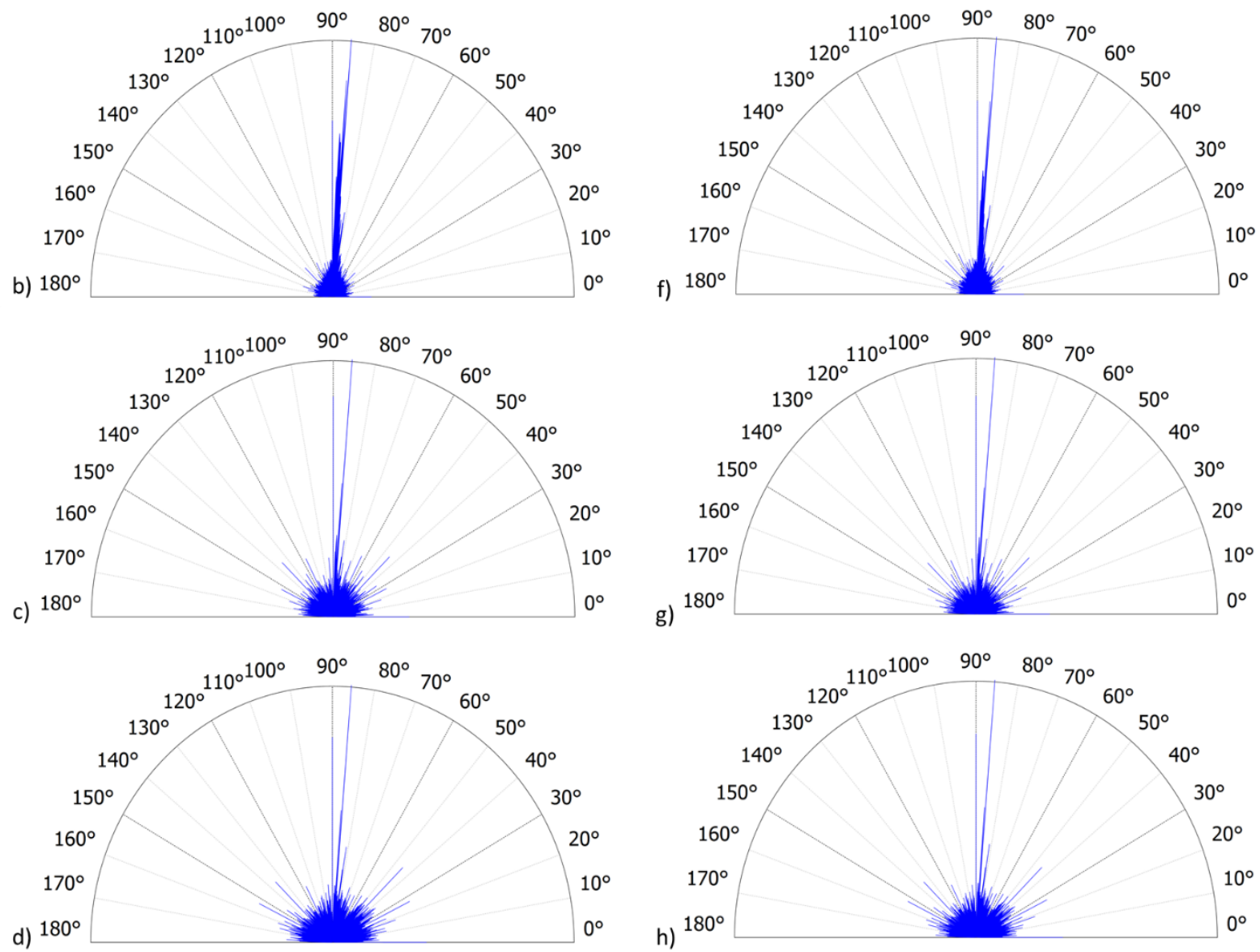

Figure 5. Surface texture direction: a) CSI SF-set; b) FV SF-set; c) XCT1 SF-set; d) XCT 2 SF-set; e) CSI SL-set; f) FV SL-set; g) XCT1 SL-set; h) XCT 2 SL-set

Further information about the SF and SL surfaces can be provided by analysis of the averaged power spectral densities (APSDs, shown in figure 6 and 7) of the surfaces. APSDs are used across a surface metrology to identify the spatial frequencies present in a surface dataset [27], and are compared here to assess the ability of XCT to identify similar spatial frequencies to those present in optical data. In the plots, the $y$ axes have been truncated to $2.5 \mu \mathrm{m}^{2}$ to allow a better visualisation of the smaller peaks at longer wavelengths. Truncation results in a loss of visualisation of the largest peak between $0.00 \mathrm{~mm}$ and $0.10 \mathrm{~mm}$, but these peaks are typically a combination of smaller scale features and high-spatial frequency noise, and of lesser interest than other visible peaks in this case. Generated APSDs are all similar; each demonstrating a similar representation of the relevant topography spatial frequencies. Peaks can be observed corresponding to the main periodic features to be expected in a LPBF surface (e.g. weld tracks, represented by three peaks between $0.10 \mathrm{~mm}$ and $0.15 \mathrm{~mm}$ wavelengths). Minor 
discrepancies in peak heights are seen between measurement instruments, but all peaks can be seen in all datasets for both the SF and SL case, at similar magnitudes.

Examination of plots, such as the texture direction and APSD graphs, and more importantly, the similarities between those plots, further demonstrates the ability of XCT for surface assessment; beyond the generation of summative visual information and surface texture parameters.

a)
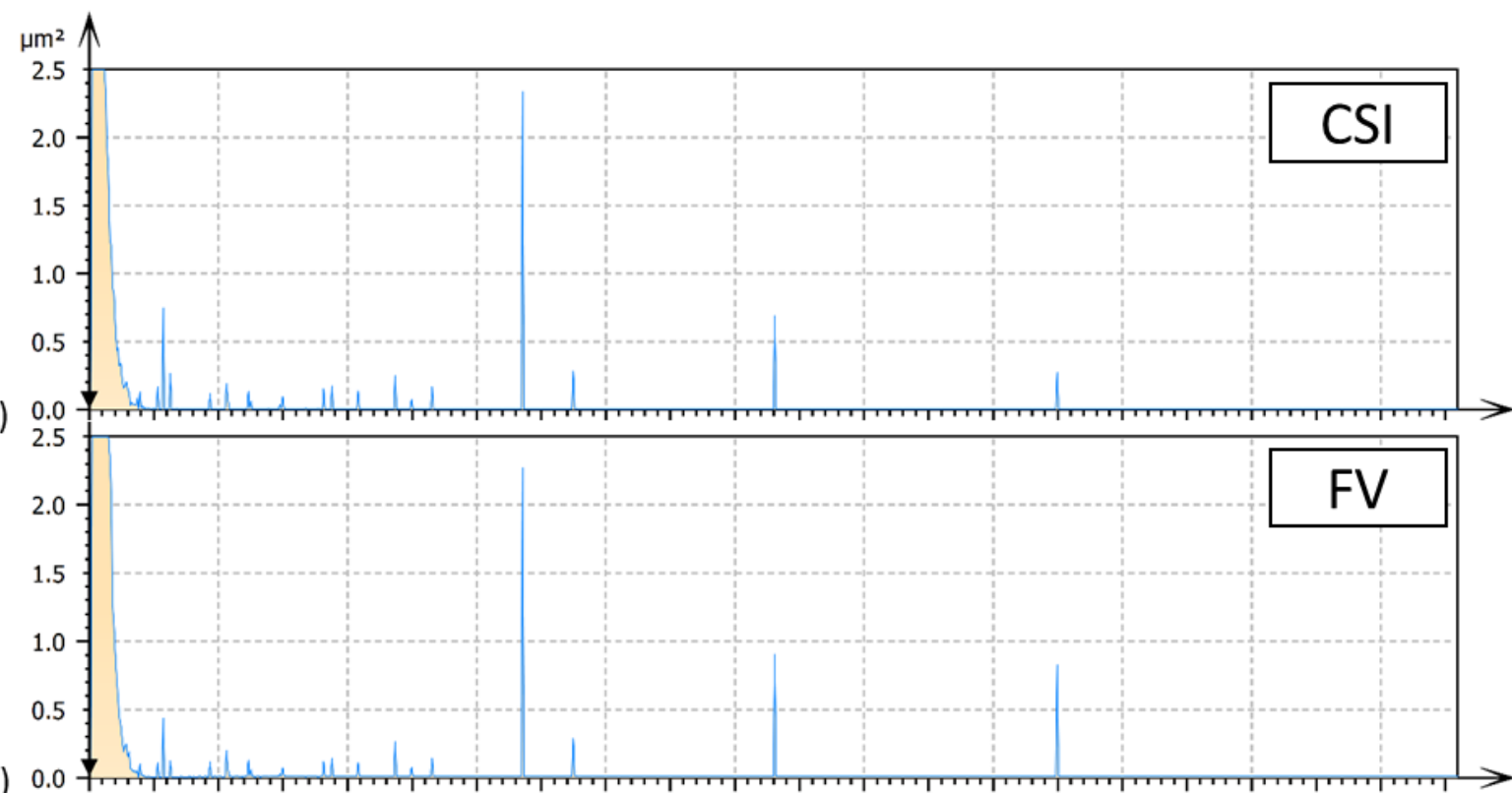

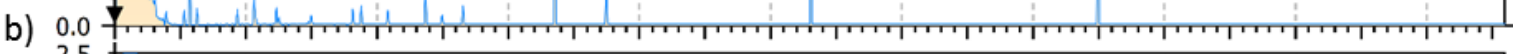

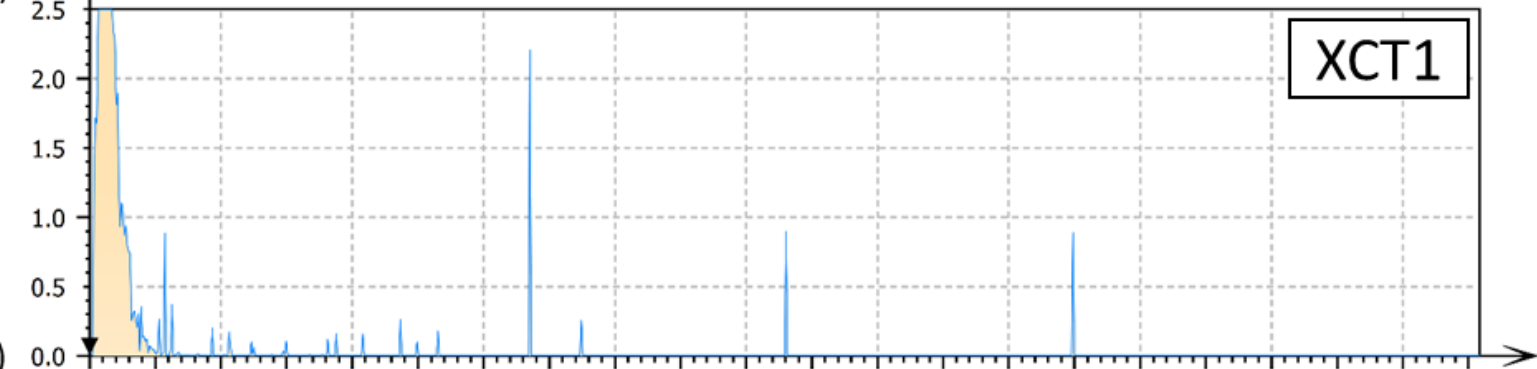

C

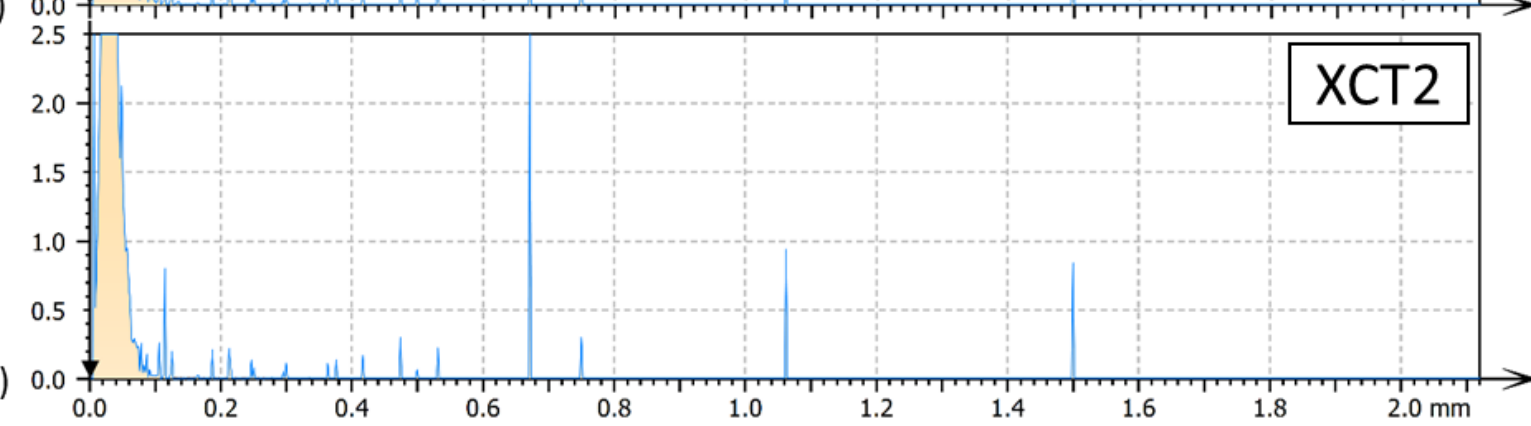

Figure 6. Averaged power spectral densities of SF-sets: a) CSI 2; b) FV; c) XCT 1; d) XCT 2. 


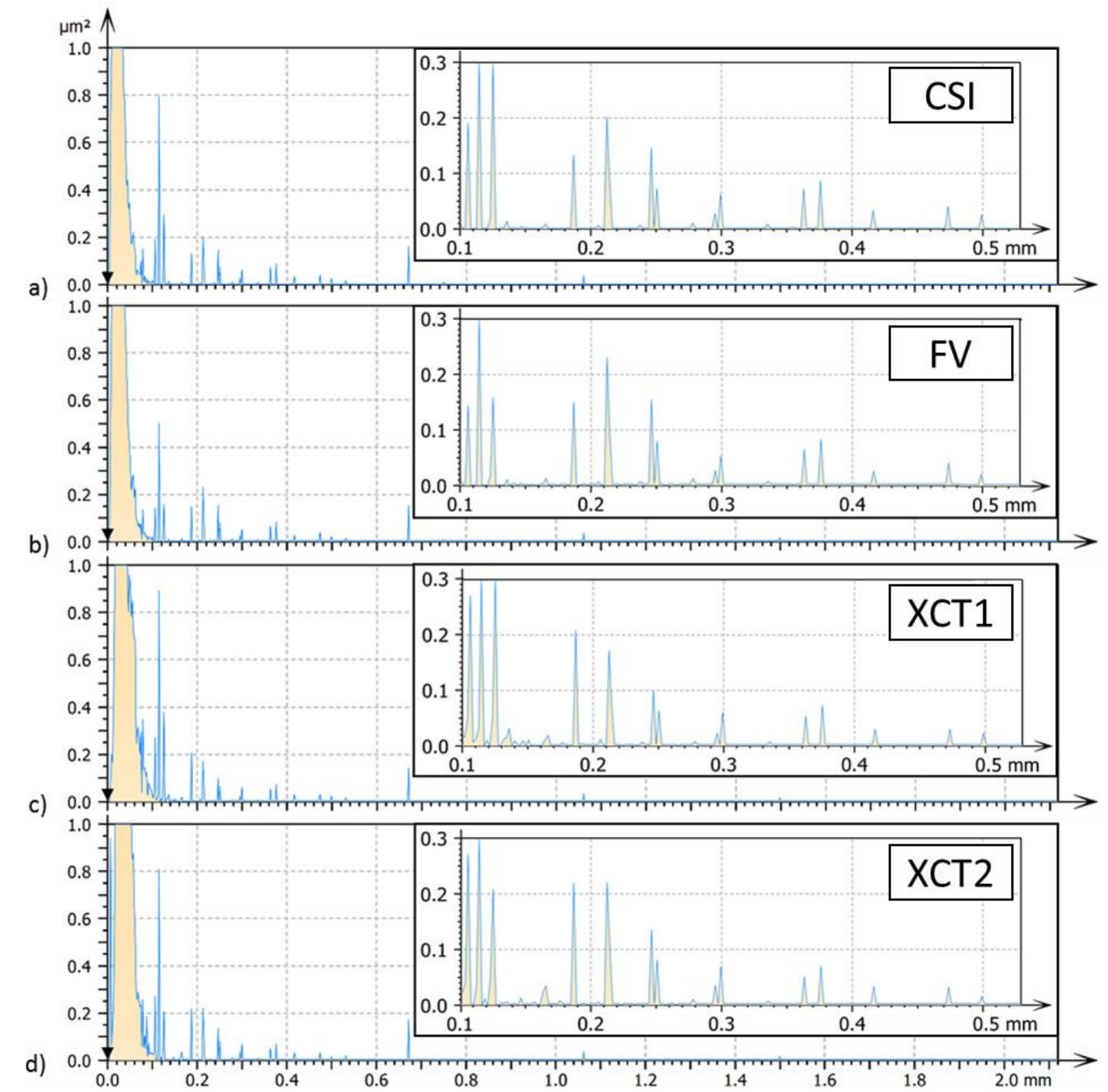

Figure 7. Averaged power spectral densities of SL-sets: a) CSI 2; b) FV; c) XCT 1; d) XCT 2. Insets are sections of the same data further truncated for visualisation along both the $x$ and $y$ axes.

\section{Conclusions and future work}

In this work, comparison between data acquired using different surface topography measurement instruments has been facilitated by accurate alignment and similar cropping of surface datasets. Visual comparison of data acquired using different surface texture measurement instruments shows notable similarities between all datasets, with the two optical systems showing the most similarity; as would have been expected. Data acquired using the two XCT systems are also visibly similar to the data produced by the two optical systems. Qualitative comparison of areal parameters calculated for aligned SF and SL surfaces showed similarity between values extracted from XCT and optical data. It is clear, therefore, that XCT technology is capable of acquiring a range of information used in surface analysis, and is able to facilitate verification of parts featuring internal or otherwise difficult-to-access surfaces. This information is particularly applicable to the metal AM industry, because of the requirement for new 
validation techniques of complex parts, as well as for non-destructive assessment of metal AM parts during process development. These analyses build on work presented previously by Townsend et al. $[12,15]$ and Thompson et al. [14], with further examination into the information that can be acquired using XCT instruments.

In this work, key areas which may play important roles in future applications of XCT for AM surface measurement have been identified. Primarily, this study was based upon analyses of single measurements, and significant work is yet to be performed in statistical testing of the methods used here in terms of measurement uncertainty. The authors have begun to address this issue in previous work [14], through development of robust statistical models of measured topographies, but many issues remain, particularly in relation to modelling of spatial correlation between individual data points within a measurement.

Additionally, algorithmic processing used during the XCT measurement process (such as during surface determination), shows that errors are easily introduced into the characterisation pipeline, despite following common good practice, occasionally failing to capture features present on surfaces, such as exogenous particles on an LPBF surface. XCT measurement of topography should, therefore, be handled with great care, as results may be unreliable, and expert assessment and interpretation of results is still required. In terms of specific surface parameters, some are clearly more robust than others between technologies, and the aforementioned measurement errors can greatly affect some outputted texture parameters. It is clear that XCT has the potential to become a viable method of surface topography measurement, but performance may be strongly dependent on the specific measurement methodology, given the sheer number of variable choices required for an XCT measurement.

A note should also be added regarding the wider applicability of these findings to the general problem of using XCT to measure internal or otherwise difficult-to-access AM surfaces. In this work, a comparison has been made involving a flat surface, though it is often the case that internal AM surfaces take complex freeform geometries (e.g. lattice structures). Analysis of such surfaces should be feasible, provided suitable steps are taken to identify and remove the underlying local shape of the part (referred to as the form component in surface metrology), so that the analysis can be focussed on smaller-scale topography. Identification and removal of form when complex shapes are involved is a challenge which has been studied for a number of years [28], but there is little work specifically regarding AM and the intrinsic complexity of freeforms and lattice structures.

Finally, in order to allow for the use of XCT for surface qualification in an industrial setting, a number of barriers remain. In terms of the XCT technology, there is a significant limit on part size and material, with X-ray penetration and resolution requirements currently preventing measurements of metal parts larger than a few centimetres in size. In regards to the metrology, methods of performing an appropriate uncertainty analysis for these measurements are not yet clear, and so great efforts must yet be made in order to attain traceable surface qualification. To address the latter issue, a rigorous sensitivity assessment of the minimum requirements of an XCT system used for surface topography applications is required. Variables that should be examined include geometric magnification, sampling strategies within the measurement, sample material, image contrast and many other control parameters currently affecting the XCT measurement process pipeline.

\section{Acknowledgements}

AT, LK and RKL would like to thank the EPSRC (grants EP/M008983/1 and EP/L01534X/1), 3TRPD Ltd. and Nikon Metrology for funding this work. NS and RKL would also like to thank the EC for supporting this work through the grant FP7-PEOPLE-MC 624770 METROSURF. The authors would 
like to thank Martin Corfield for XCT 2 data acquisition and Luke Hunter for XCT 2 data reconstruction, as well as Digital Surf for providing the MountainsMap software.

\section{References}

[1] I. Gibson, D.W. Rosen, B. Stucker, Additive manufacturing technologies: 3D printing, rapid prototyping, and direct digital manufacturing, Springer, New York, 2014.

[2] G. Ameta, R. Lipman, S. Moylan, P. Witherell, Investigating the role of geometric dimensioning and tolerancing in additive manufacturing, J. Mech. Des. 137 (2015) 111401.

[3] R.K. Leach, Metrology for additive manufacturing, Meas. + Control. 49 (2016) 132-135.

[4] P.I. Stavroulakis, R.K. Leach, Review of post-process optical form metrology for industrial-grade metal additive manufactured parts, Rev. Sci. Instrum. 87 (2016) 41101.

[5] A. Townsend, N. Senin, L. Blunt, R.K. Leach, J.S. Taylor, Surface texture metrology for metal additive manufacturing: a review, Precis. Eng. 46 (2016) 34-47.

[6] L. De Chiffre, S. Carmignato, J.-P. Kruth, R. Schmitt, A. Weckenmann, Industrial applications of computed tomography, Ann. CIRP 63 (2014) 655-677.

[7] ISO/CD 10360-11: Geometrical product specification (GPS) -- acceptance and reverification tests for coordinate measuring machines (CMM) - Part 11: Computed tomography. (Under development, International Organisation for Standardisation)

[8] A. Thompson, I. Maskery, R.K. Leach, X-ray computed tomography for additive manufacturing: a review, Meas. Sci. Technol. 27 (2016) 72001.

[9] G. Pyka, G. Kerckhofs, A. Braem, T. Mattheys, J. Schrooten, M. Wevers, Novel micro-ct based characterization tool for surface roughness measurements of porous structures, in: SkyScan User Meeting, Mechelen, (2010) 1-5.

[10] G. Kerckhofs, G. Pyka, M. Moesen, S. Van Bael, J. Schrooten, M. Wevers, High-resolution microfocus x-ray computed tomography for 3D surface roughness measurements of additive manufactured porous materials, Adv. Eng. Mater. 15 (2013) 153-158.

[11] A. Townsend, L. Pagani, P. Scott, L.A. Blunt, Areal surface texture data extraction from x-ray computed tomography reconstructions of metal additively manufactured parts, Precis. Eng. 48 (2017) 254-264.

[12] A. Townsend, L. Pagani, L. Blunt, P.J. Scott, X. Jiang, Factors affecting the accuracy of areal surface texture data extraction from X-ray CT, Ann. CIRP 66 (2017) 547-550.

[13] A. Townsend, R. Racasan, P. Bills, A. Thompson, N. Senin, R. Leach, L. Blunt, Results from an interlaboratory comparison of areal surface texture parameter extraction from X-ray computed tomography of additively manufactured parts, euspen's 17th Int. Conf. Exhib., Hannover, Germany, (2017).

[14] A. Thompson, N. Senin, C. Giusca, R. Leach, Topography of selectively laser melted surfaces: a comparison of different measurement methods, Ann. CIRP 66 (2017) 543-546.

[15] A. Townsend, L. Pagani, P. Scott, L.A. Blunt, Areal surface texture data extraction from X-ray computed tomography reconstructions of metal additively manufactured parts, Precis. Eng. 48 (2017) 254-264.

[16] M. Bartscher, A. Staude, K. Ehrig, A. Ramsey, The Influence of Data Filtering on Dimensional Measurements with CT, in: Conf. Industrial Computed Tomography, Wels, (2012) 16-20.

[17] Volume Graphics, VGStudio MAX. https://www.volumegraphics.com/en/products/vgstudiomax.html, 2017 (accessed 26.05.17).

[18] K. Kiekens, F. Welkenhuyzen, Y. Tan, P. Bleys, A. Voet, J.-P. Kruth, W. Dewulf, A test object with parallel grooves for calibration and accuracy assessment of industrial computed tomography (CT) metrology, Meas. Sci. Technol. 22 (2011) 115502.

[19] Visual Computing Lab - ISTI - CNR, MeshLab. http://meshlab.sourceforge.net/, 2017 (accessed 26.05.17).

[20] I. Jolliffe, Principal Component Analysis, in: Wiley StatsRef Online, Wiley, New York, 2014. 
[21] Digital Surf, Mountains ${ }^{\circledR}$ surface imaging \& metrology software. http://www.digitalsurf.com/en/mntpremium.html, 2017 (accessed 26.05.17).

[22] J. Condeço, L.H. Christensen, B.G. Rosén, Software relocation of 3D surface topography measurements, Int. J. Mach. Tools Manuf. 41 (2001) 2095-2101.

[23] R.K. Leach, H. Haitjema, Bandwidth characteristics and comparisons of surface texture measuring instruments, Meas. Sci. Technol. 21 (2010) 32001.

[24] ISO 25178-2: 2012, Geometrical product specifications (GPS) -- surface texture: areal -- part 2: terms, definitions and surface texture parameters. (International Organisation for Standardisation)

[25] R.K. Leach, Characterisation of areal surface texture, Springer-Verlag, Berlin, 2013.

[26] N. Senin, A. Thompson, R.K. Leach, Characterisation of the topography of metal additive surface features with different measurement technologies, Meas. Sci. Technol. 9 (2017) 095003

[27] T.D.B Jacobs, T. Junge, L. Pastewka, Quantitative characterization of surface topography using spectral analysis, Surf. Topogr.: Metrol. Prop. 5 (2017) 013001

[28] X. Jiang, P. Scott, D. Whitehouse, Freeform surface characterisation - a fresh strategy, Ann. CIRP 56 (2007) 553-556 